\title{
Integration and workflow: Their impact on ROI in broadcast media asset management projects
}

\begin{abstract}
Jeremy Bancroft
is Managing Director of Media Systems Consulting Limited, a broadcast consultancy practice specializing in file-based broadcast operations, content tracking, digital rights management, workflow management and business process improvement. $\mathrm{He}$ is also a non-executive director of Blue Order, and has been responsible for the worldwide strategic marketing of digital asset management solutions to broadcasters. As a founder director of OmniBus Systems, and President of its US subsidiary, he designed, sold and implemented a number of innovative news and transmission playout solutions to customers including NRK Norway, APTN London and TV6 Russia. Jeremy started his career as a hardware and software design engineer. He has presented papers at SMPTE, SATIS Paris, IBC Amsterdam, NewsWorld Barcelona, ETA Pasadena, Content World San Jose, Broadcast Asia Singapore and at the European Broadcasting Union in Geneva. He has also been an active industry participant in the EBU Digital Rights Management Technology (DRM-T) committee.
\end{abstract}

Keywords: broadcast, MAM, workflow integration, legacy systems, ROI, collaborative workflows

Abstract For broadcasters migrating from traditional linear tape-based operations to non-linear file-based workflows, media asset management (MAM) has a key role to play. By enabling widespread, simultaneous access to media, and by supporting collaborative workflows, MAM provides an essential tool to broadcasters in the digital age. However, many such projects have failed to fully deliver the benefits promised by the vendor, and anticipated by the broadcaster. The author proposes that there are two key indicators of future success in broadcast MAM projects: Understanding the customers' specific workflows, and integrating with the customers' legacy systems. This paper explores this proposition, and provides case study evidence to support the claims.

Jeremy Bancroft Managing Director, Media Systems Consulting Ltd, 27 Middle Street, Croxton Kerrial, Grantham, Lincs NG23 1OP, UK Tel: +44 (0)7970 134335 e-mail: jeremy@mscl.net

\section{INTRODUCTION}

Broadcast is a highly specialized area. It is unlike any other industry in the way that it employs large numbers of separate systems and has very complex and collaborative workflows.

A typical broadcast enterprise comprises separate islands of operation with little or no integration between them. There are disparate management information systems and often multiple repositories of metadata.

Information is often manually keyed into multiple systems and media is transferred on tape using "sneaker net" within the facility, and on tape using FedEx to other, outside parties.

Over the past few years, we have started to see a number of pioneering organizations taking significant steps to change this by deploying "file-based" solutions, with media asset management (MAM) at their core. Great savings were promised and more streamlined operations were expected.

Those of you who have read Geoffrey 
A. Moore's definitive work, Crossing The Chasm, may recognize these organizations as "early adopters". Others would call them pioneers. You know how to recognize a pioneer, right? They're the ones with arrows in their backs!

A number of early adopters within the broadcast sector have deployed "MAMcentric, file-based" solutions, with varying degrees of success. Several have been severely disappointed and have failed to achieve a return on investment (ROI) (and some never will).

We believe that the success of these projects is heavily influenced by two factors:

1. The organization's understanding of its workflows; and

2. The degree of integration between the new MAM solution and the other systems that exist within the broadcast operation.

\section{WORKFLOWS}

We strongly recommend that any broadcast organization considering MAM should start by looking closely at the workflows.

First, you need to understand the current workflows. In our experience, very few broadcast operations really understand their existing workflows. They often think that they understand them, and they may understand fragments in minute detail, but it is vital to understand the end-to-end workflow. Without this, it is extremely likely that any new solution you deploy, or commission someone to deploy on your behalf, is going to fail to deliver the benefits you hoped for, simply because some essential parts of your existing workflow are not replicated or replaced.

Secondly, model the workflow that you want to achieve and again, clearly document it, and preferably draw it.

This is an important step, as it will ensure that you have replaced, replicated or improved all the current processes before designing a solution that will successfully streamline your operations and remove cost.

\section{Why do I need to understand workflows that I am replacing?}

Now, you could take the position that:

"I am designing a new system, and it will introduce new workflows that replace the old ones. So why do I need to spend time and money understanding what we do now, because I know it's inefficient?"

The simple answer is that, in our experience, there will almost certainly be parts of your operation that totally rely upon some manual functions, for example to exchange vital information or deal with exceptions. These functions usually exist because they are needed. What's more, they are only known by one or two people in the organization.

\section{How do I do that?}

Capture these workflows. Document and draw them. Distribute the document, or sections of it, to existing operations staff and ensure that they agree that it represents all the current workflows. You will also find that by involving these operations staff early in the process, it will help with the acceptance of new systems, technology and processes. This is partly because the operations staff will now know that those planning the new system understand what it is that they do.

In order to capture the workflows, 
you can use internal resources, but if you do:

- Ensure that this is their sole task for as long as it takes. It is far too important a task to expect someone to do this alongside their day job. Something is going to suffer.

- Ensure that the person or persons you have selected have demonstrated their ability to understand and clearly document complex workflows, and that they have sufficient domain experience (ie they understand broadcast operations) to know when what they are being told is incomplete or just plain wrong.

\section{Technology is not always the (only) answer}

You may well find that just by understanding the current workflows, you can identify substantial savings that can be brought about just by changing operational practices and procedures. It may not be necessary to rely on substantial deployments of technology.

\section{Case study 1}

By way of an example, we were recently approached by a large broadcaster in the Middle East who wanted to deploy a file-based workflow with MAM.

The chief engineer had already started selecting technology because he believed that file-based workflows supported by MAM would provide substantial savings to the business. He had notionally allocated a budget of almost \$2-3m for the project and wanted to move quickly. He still had to convince his general manager that this was a good investment, however.

The chief engineer approached us to talk about technology and we advised that he first undertake a workflow study that would identify the priority areas within his business, where deployment of new technology would have the greatest impact.

Blue Order was commissioned to perform this workflow study and two of us traveled to site to spend a week with the management team and operations staff.

By the end of the first day, it was obvious to us where the real opportunities for quick-wins existed. We identified that by making simple changes to the way they managed the subtitling of European American movies and episodic programming, the broadcaster could save almost $\$ 1.5 \mathrm{~m}$ per year.

We also proved that by deploying file-based workflows; the organization could save over $\$ 1 \mathrm{~m}$ per year in tape and video tape recorder (VTR) maintenance costs alone.

But the key issue was that with some changes in working practices, the organization could save a further $\$ 1-2 \mathrm{~m}$ per year in tape stock, space and very importantly, staff turnover costs.

We documented our findings and returned to site to present them to the senior management team. Of course, there was some skepticism from managers about changes in their own departments, but strangely enough, they all agreed about the how the changes to each other's parts of the organization would improve their business.

The chief engineer now has his budget, and his general manager and CFO have quantitative and verifiable evidence of the ROI of this project.

They are starting with a change in subtitling processes, which has an ROI of less than one month! 


\section{INTEGRATION}

The second indicator of future success is integration.

A typical broadcast operation has multiple management information systems and several repositories of metadata. These systems rarely link up; instead, the same data must be manually entered and updated in multiple systems.

When planning a new approach, it is important to decide which systems will stay and which systems will be replaced, and to decide which metadata system will be "master".

This should always be the MAM system, right?

Well, not necessarily.

Most broadcast operations have a channel management system. This system is often responsible for the commissioning, licensing and scheduling of programs, and may also be responsible for the sales and placement of commercials and sponsorship messages.

This system will almost inevitably have, or interface with, financial accounting systems and may well have a tape library management module.

Because this system touches so many parts of the broadcaster's business, it is very difficult to change. Entire departments often grow around just supporting channel management.

Usually, the program acquisition department knows about the impending arrival of media before anyone else. And guess which system they use - the channel management system.

Planning schedules, or grids, are often created by the scheduling department 36 months before a program is due to transmission. And guess which system they use - right again, the channel management system.
So, given that the channel management system contains information (metadata) often long before anything else, and often contains business related information on commercial spot sales, why not make it "master?"

Information may be derived from channel management to populate the MAM system and drive any workflows. It is also likely that information collected in the MAM system would be useful to channel management users, so better make this a two-way interface. But business-related changes will almost always happen first in channel management.

Production schedules are another area where different systems produce outputs that fundamentally affect workflows. Wouldn't it be great if my creative services department had task lists pop up on their craft-edit platform?

Point-to-point integration between the production scheduling system and the edit system might provide this functionality, but if I am planning resources, or monitoring throughput, how do I interrogate this? This is another area where a connected MAM system can provide a portal through which task lists can be distributed, workflows can be monitored and throughput can be reported.

\section{Case study 2}

Integration with legacy databases, such as existing library management systems, is often a requirement for Blue Order's customers. ZDF, arguably Europe's largest public broadcaster, had a legacy system called Fernseh Data Base (FDB) which was supporting thousands of concurrent users on a terminal server based system. 
The FDB system was not capable of supporting integration with production and newsroom systems, nor was it able to provide video browsing functionality.

So ZDF approached Blue Order to provide a new solution that would leave FDB in place for many of the users, but would provide additional functionality for up to 800 "super-users" with integration across production and newsroom platforms.

This way, it was not necessary to incur the expense of totally replacing a solution that still met the needs of the majority of users.

ZDF also benefited from the streamlined workflows that could be achieved with the integration available with Blue Order's MAM solution.

\section{But what about "out of the box"?}

One of the real challenges for MAM vendors (and their customers) is the trade-off between productized offerings and customized solutions.

On the one hand, broadcasters want to buy productized systems that are cheaper and faster to deploy, and by the nature of the fact that they are a "product" they are easier for the vendor to support (and hopefully this is reflected in the outrageous support fees being charged).

Unless the solution can offer a high degree of integration with other systems in use at the broadcaster's facility, however, experience suggests that there is very little chance of a realistic ROI. This is an important point to note.

No integration. No ROI. Period.

And by and large, each broadcaster has a unique combination of systems and workflows that are both difficult and costly to integrate on a case-by-case basis.

This challenge can be addressed by a modularized solution with a core set of available, price-listed integrations, and a full set of simple object access protocol application programming interfaces (SOAP APIs) that allow for straightforward integration into the more esoteric sub-systems that abound in the broadcast space.

Instead of the system being the product, the modules are the products. The modules are combined in a unique way to create the system

Microsoft's Connected Services Framework (CSF) is an excellent step forward in this area and provides a standardized method of integration between vendors who support this initiative.

Blue Order has announced its support for CSF and expects to be deploying its first solutions using this method of integration later in 2005.

\section{So now we have workflow and integration, what's next?}

In a word: data model (OK, that's two words).

The development of a complex, custom data model is expensive, and may represent a significant proportion of the overall project budget. Blue Order customers who have understood the core importance of having the right data model have tended to spend a lot of time and money developing their own data model (often with the help of thirdparty consulting organizations).

The ideal data model for a broadcast MAM solution will encompass many different types of metadata. Technical metadata, descriptive metadata, broadcast metadata, usage metadata, rights metadata. Each of these types of metadata, and their relationship to the media asset, needs to be managed. This indicates the requirement for an entity 
relationship model (or ERM) database.

Blue Order has spent many years working with, and refining broadcast data models and has published a white paper entitled "The Generalized Video Data Model (or GVDM)". This is a data model that encompasses all the key types and fields of metadata and establishes their interrelationships. It is totally configurable and we haven't come across a single customer requirement that cannot be met using this framework.

As we have considerable experience of audio archive projects, we have also published the GADM which is, as you might expect, the Generalized Audio Data Model.

\section{Case study 3}

When Swiss Radio needed a MAM system to manage their library of literally millions of audio clips and associated metadata, spread across multiple sites, they commissioned Blue Order to develop a data model and MAM solution that would meet their specific needs.

Surprisingly, audio data models tend to be far more complex than their video counterparts, with separate subclassifications and data sets for classical music, popular music, spoken word and sound effects.
At the time, this was perhaps the most complex audio library project in the world, with very specific cataloguing and workflow requirements. We considered that the GADM would be put through its paces within this project, but it still proved to have the flexibility and extensibility to address this demanding customer's requirements.

\section{CONCLUSION}

My prediction is that in the broadcast world, 2005 will be known as the beginning of the file-based era.

We have seen an unprecedented number of broadcasters planning projects that could radically change their operational and business processes. A number of these projects will probably be hugely successful. But then, a number will probably fail to produce the benefits expected. There will be no ROI.

It is our belief that many of those failures could be prevented by engaging individuals and organizations who know this space, who have experience of similar projects (both successful and otherwise), by understanding current and planned workflows, and by ensuring that the solutions to be deployed will be fully integrated with the rest of the broadcast infrastructure. 